\title{
A LOWER BOUND FOR THE NUMBER OF ZEROS OF A MEROMORPHIC FUNCTION AND ITS SECOND DERIVATIVE
}

\author{
by J. K. LANGLEY
}

(Received 13th September 1994)

We prove that for a function $f(z)$ transcendental and meromorphic in the plane and not of the form $\exp (a z+b)$, we have either $N\left(r, 1 / f f^{\prime \prime}\right) \neq o\left(T\left(r, f^{\prime} / f\right)\right)$ or $\varlimsup_{r \rightarrow \infty} \frac{\log N\left(r, 1 / f f^{\prime \prime}\right)}{\log \log r} \geqq 2$.

1991 Mathematics subject classification: 30D35.

\section{Introduction}

The following theorem was proved in [12], confirming a conjecture of Hayman [8].

Theorem A. Suppose that $f$ is meromorphic in the plane such that $f$ and $F$ have only finitely many zeros, where $F=f^{\prime \prime}+a_{1} f^{\prime}+a_{0} f$ and the $a_{j}$ are rational functions with $a_{j}(z)=O\left(|z|^{j-2}\right)$ as $z \rightarrow \infty$. Then $f^{\prime} / f$ is rational. In particular, if $f$ and $f^{\prime \prime}$ have no zeros, we have $f(z)=\exp (a z+b)$ or $f(z)=(a z+b)^{-n}$ with $a$ and $b$ constants and $n$ a positive integer.

The proof of Theorem $\mathrm{A}$ in [12] begins by using a device of Frank $[5,6,7]$. If $f_{1}, f_{2}$ are linearly independent solutions of the associated homogeneous equation $w^{\prime \prime}+a_{1} w^{\prime}+$ $a_{0} w=0$, then we define $g$ by $g^{2}=f / F$, and the functions $w_{j}=f_{j}^{\prime} g-f_{j}\left(f^{\prime} / f\right) g$ solve an equation $w^{\prime \prime}+a_{1} w^{\prime}+b w=0$ in which $b$ is meromorphic with only finitely many poles. The paper [15] uses this method to determine all functions $f$ meromorphic in the plane for which $f$ and $F$ have only finitely many zeros, with the $a_{j}$ any rational functions. In the particular case $F=f^{\prime \prime}$, an alternative, but related, approach, used by Mues in [16], is to write $H=z-f / f^{\prime}$ so that $H$ has only finitely many multiple points and its Schwarzian derivative $\{H, z\}=H^{(3)} / H^{\prime}-\frac{3}{2}\left(H^{\prime \prime} / H^{\prime}\right)^{2}[10,11]$ has only finitely many poles. Using the modified auxiliary function $G=z-h f / f^{\prime}$, with $h$ a constant, Bergweiler proved the following in [2].

Theorem B. Suppose that $a$ is a constant such that $a \neq 1$ and $1 /(a-1)$ is not a positive integer, and suppose that $f$ is transcendental and meromorphic of finite order in the plane such that $L=f f^{\prime \prime}-a f^{\prime 2}$ has only finitely many zeros. Then 


$$
f(z)=\exp (A z+B), \quad A, B \in \mathbb{C} .
$$

It is necessary to assume in Theorem $B$ that $a$ is not of the form $(n+1) / n$, with $n$ a positive integer, because of the example $f(z)=g(z)^{-n}$, with $g$ any entire function such that $g^{\prime \prime}$ has only finitely many zeros. The case $a=1$ must also be excluded, because of examples such as $f(z)=\cos z, f(z)=\left(1+\mathrm{e}^{z}\right)^{n}(n \leqq 1), f(z)=\mathrm{e}^{g(z)}, g^{\prime \prime}(z) \neq 0$ (see also [17]). Bergweiler's proof in [2] does not use the Schwarzian derivative, but proceeds by applying the main result of [3] to show that the inverse function of $G$ has only finitely many singularities, this leading to an estimate for $G^{\prime}(z)$ at fixpoints of $G$.

In the present paper, we remove the order restriction in Theorem $B$, and strengthen the conclusion that if (1.1) does not hold then $L$ must have infinitely many zeros, thus also improving Theorem $\mathrm{A}$ in the most important case $F=f^{\prime \prime}$. We state the following result, part (i) of which was proved by Frank and Hellerstein in [6], with part (ii) appearing in [14].

Theorem C. Suppose that $f$ is transcendental meromorphic in the plane and $N\left(r, 1 / f f^{(k)}\right)=o\left(T\left(r, f^{\prime} / f\right)\right)$ for some $k \geqq 2$. (i) If $k \geqq 3$ then (1.1) holds. (ii) The same conclusion holds if $k=2$ and $f$ has finite lower order.

It seems possible that Theorem $\mathrm{C}$ holds without the order restriction in part (ii). The proof in [14] uses the auxiliary function $H=z-f / f^{\prime}$ and the following combination of results of Shea [18] and Eremenko [4]. If $H$ is transcendental and meromorphic of finite lower order in the plane, and the counting function

$$
N_{1}(r, H)=N\left(r, 1 / H^{\prime}\right)+N(r, H)-\bar{N}(r, H)
$$

of the multiple points of $H$ satisfies $N_{1}(r, H)=o(T(r, H))$, and if $H$ is normalised so that $\delta(\infty, H)=0$, then the order $\rho$ of $H$ is such that $2 \rho$ is an integer not less than two, and there are slowly varying functions $L_{j}(r)$ such that

$$
-\log \left|H^{\prime}\left(r \mathrm{e}^{i \theta}\right)\right|=\pi r^{\rho} L_{1}(r)\left(\left|\cos \left(\rho\left(\theta-L_{2}(r)\right)\right)\right|+o(1)\right)
$$

outside an exceptional set. With the hypotheses of Theorem $D$, part (ii), the assumption that $H$ is transcendental and (1.2) lead to an upper estimate for $m(r, 1 / f)$, which in turn contradicts the fact that $N(r, 1 / f)$ is small. This approach does not seem to work for the problem considered in Theorem $\mathrm{B}$, and (1.2) is not available when $H$ has infinite order. We prove here the following theorem.

Theorem 1. Suppose that $f$ is transcendental and meromorphic in the plane, and that $F=f f^{\prime \prime}-a f^{\prime 2}, a \in \mathbb{C}$.

(i) If $a \neq 1$ and $1 /(a-1)$ is not a positive integer and

$$
N(r)=o\left(T\left(r, f^{\prime} / f\right)\right) \text { and } \varlimsup_{r \rightarrow \infty} \frac{\log N(r)}{\log \log r}<2
$$


holds with $N(r)=N_{2}(r)=N(r, 1 / F)$, then (1.1) holds.

(ii) Suppose that $a \neq 1$ and $1 /(a-1)$ is not an integer, or that $a=0$. If (1.3) holds with $N(r)=N_{3}(r)$, where $N_{3}(r)$ counts the zeros of $F$ which are not multiple zeros of $f$, then (1.1) holds.

In particular, if $N(r)$ counts the simple zeros of $f$ and the zeros of $f^{\prime \prime}$ which are not multiple zeros of $f$, and (1.3) holds, then we have (1.1). Theorem 1 will be proved using the following auxiliary result.

Theorem 2. Suppose that $H$ is transcendental and meromorphic in the plane of order $\rho(H)$ such that

$$
N_{1}(r, H)=o(T(r, H)), \varlimsup_{r \rightarrow \infty} \frac{\log N_{1}(r, H)}{\log \log r}<2,
$$

and suppose further that $q$ is a constant with $q<\rho(H)$. Then $H$ has fixpoints $z$ with $|z|$ arbitrarily large and $\left|H^{\prime}(z)\right|>|z|^{q}$.

We make some remarks about Theorem 2. First, we are free to assume that $\rho(H)$ is positive, by the results of Shea and Eremenko already cited. Further, the first inequality of (1.4) implies that $H$ has infinitely many fixpoints (as noted in [2]) and, if $H$ has finite lower order, implies additionally that (1.2) holds. However, these fixpoints may and almost certainly must lie in regions where the cosine term in (1.2) is small.

Of course, if $f^{\prime} / f$ is not of small growth then Theorem 1 gives only a weak estimate for the number of zeros of $f$ and $f^{\prime \prime}$. We indicate in Section 5 where the second inequality of (1.4) seems to be necessary for our method, and in Section 8 we outline the proof of a stronger result which holds when $f^{\prime} / f$ has very large growth.

\section{Preliminaries}

We begin by summarizing some facts from the Wiman-Valiron theory [9]. If $\gamma>1 / 2$ is a constant and $g(z)=\sum_{k=0}^{\infty} a_{k} z^{k}$ is a transcendental entire function with maximum modulus $M(r, g)$ on $|z|=r>1$, the central index $v(r, g)$ is the largest $n$ such that $\left|a_{n}\right| r^{n}=\mu(r, g)=\max _{m}\left|a_{m}\right| r^{m}$, and there is a set of normal $r$, the complement of this set having finite logarithmic measure, such that if $r$ is normal and $\left|z_{0}\right|=r$ with $\left|g\left(z_{0}\right)\right|>$ $\frac{1}{2} M(r, g)$, we have

$$
\begin{gathered}
(\log M(r, g)) / 2 \log r \leqq v(r, g) \leqq(\log M(r, g))^{3 / 2}, \\
M\left(r, g^{\prime}\right) \sim M(r, g) v(r, g) / r, \text { while } g(z) \sim\left(z / z_{0}\right)^{v(r, g)} g\left(z_{0}\right),
\end{gathered}
$$

and

$$
g^{(i)}(z) / g(z) \sim(v(r, g) / z)^{j} \text { for } j=1,2 \text { and }\left|\log \left(z / z_{0}\right)\right| \leqq v(r, g)^{-\gamma}
$$

We require in addition representations in annuli for entire functions of very small 
growth. Suppose that $\delta$ and $\varepsilon$ are positive constants, and that $f_{1}, f_{2}, \ldots, f_{n-1}$ and $f_{n}=f$ are transcendental entire functions each satisfying $T\left(r, f_{j}\right)=O(\log r)^{2-\delta}$. Then if $r_{0}$ is large the number of zeros of the $f_{j}$ in $r_{0} \leqq|z| \leqq\left(r_{0}\right)^{1+\varepsilon}$ is $O\left(\log r_{0}\right)^{1-\delta}$, and we can find $r_{1}, r_{2}$ with

$$
\log r_{0} \leqq \log r_{1} \leqq \log r_{1}+\left(\log r_{0}\right)^{2 \delta / 3}=\log r_{2} \leqq(1+\varepsilon) \log r_{0}
$$

and such that none of the $f_{j}$ have zeros in

$$
\log s_{1}=\log r_{1}-\left(\log r_{0}\right)^{2 \delta / 3} \leqq \log |z| \leqq \log r_{2}+\left(\log r_{0}\right)^{2 \delta / 3}=\log s_{2}
$$

If $f(z)=\prod_{j=1}^{\infty}\left(1-z / a_{j}\right)$, assuming that $f(0)=1$, then for $r_{1} \leqq|z| \leqq r_{2}$ we can write

$$
f(z)=z^{n\left(s_{1}, 1 / f\right)} \Pi_{\left|a_{j}\right| \leqq s_{1}}\left(-1 / a_{j}\right)\left(1-a_{j} / z\right) \Pi_{\left|a_{j}\right| \geqq s_{2}}\left(1-z / a_{j}\right)
$$

and we have (see $[1,13]$ for details)

$$
\begin{gathered}
\left|\Pi_{\left|a_{j}\right| \leqq s_{1}}\left(1-a_{j} / z\right)-1\right| \leqq \exp \left(n\left(s_{1}, 1 / f\right) s_{1} / r_{1}\right)-1 \leqq \exp \left(-\left(\log r_{0}\right)^{\delta / 2}\right) \\
\left|\Pi_{\left|a_{j}\right| \geqq s_{2}}\left(1-z / a_{j}\right)-1\right| \leqq \exp \left(r_{2} \int_{s_{2}}^{\infty}(1 / t) d n(t, 1 / f)\right)-1 \\
\leqq O\left(\left(r_{2} / s_{2}\right) \log s_{2}\right) \leqq \exp \left(-\left(\log r_{0}\right)^{\delta / 2}\right)
\end{gathered}
$$

Lemma 1. There is a positive constant $c_{1}$ with the following property. Suppose that $c>0$ and $L>(1+c) s>1+c$ and $F(z)$ is analytic in the closed rectangular region $\Omega$ given by $|\operatorname{Re}(z)| \leqq 3 \pi L, L s \leqq \operatorname{Im}(z) \leqq L S \leqq 2 L s$, with $|F(z)| \leqq c L^{-2}$ there. Then the equation

$$
w^{\prime \prime}(z)+(1-F(z)) w(z)=0
$$

has solutions $U(z), V(z)$ in $\Omega$ such that, with $\left|\varepsilon_{j}(z)\right| \leqq c_{1} c s / L$,

$$
\begin{gathered}
U(z)=\mathrm{e}^{-i z}\left(1+\varepsilon_{1}(z)\right), \quad U^{\prime}(z)=-\mathrm{ie}^{-i z}\left(1+\varepsilon_{2}(z)\right), \\
V(z)=\mathrm{e}^{i z}\left(1+\varepsilon_{3}(z)\right), \quad V^{\prime}(z)=\mathrm{ie}^{i z}\left(1+\varepsilon_{4}(z)\right) .
\end{gathered}
$$

Proof. This is similar to Lemma 1 of [12], but with a different region $\Omega$. We choose a solution $v$ of the equation $v^{\prime \prime}+2 i v^{\prime}-F v=0$ such that $v(X)=1, v^{\prime}(X)=0$, where $X=\mathrm{i} L S$. Differentiating twice shows that

$$
v(z)=1-(1 / 2 i) \int_{X}^{z}\left(\mathrm{e}^{2 \mathrm{i}(t-z)}-1\right) F(t) v(t) d t, v^{\prime}(z)=\int_{X}^{z} \mathrm{e}^{2 \mathrm{i}(t-z)} F(t) v(t) d t
$$

We take as path of integration the straight line segment from $X$ to $\operatorname{Re}(z)+\mathrm{i} L S$ followed by that from $\operatorname{Re}(z)+\mathrm{i} L S$ to $z$. If $d s$ denotes arc-length on this path, (2.3) gives 


$$
|v(z)-1| \leqq \int_{x}^{z}|F(t) v(t)| d s
$$

We set

$$
W(\zeta)=\log \left(1+\int_{X}^{\zeta}|F(t) v(t)| d s\right)
$$

with $\zeta$ lying on the above path. Then $d W / d s \leqq|F(\zeta)|$, so that using $d_{1}, d_{2}, \ldots$ to denote positive constants not depending on $c$, we obtain $W(z) \leqq \int_{X}^{z}|F(t)| d s \leqq d_{1} c s L^{-1}$. Thus

$$
|v(z)-1| \leqq \exp \left(d_{1} c s L^{-1}\right)-1 \leqq d_{2} c s L^{-1} \leqq d_{3},\left|v^{\prime}(z)\right| \leqq \int_{X}^{z}|F(t)| d_{3} d s \leqq d_{4} c s L^{-1}
$$

Now we set $V(z)=\mathrm{e}^{\mathrm{i} z} v(z)$ so that $V$ solves (2.1) and (2.2) follows at once. To form $\boldsymbol{U}$, choose a solution $u$ of $u^{\prime \prime}-2 i u^{\prime}-F u=0$, such that $u(Y)=1, u^{\prime}(Y)=0$, where $Y=\mathrm{i} L s$. The integral equation for $u$ is $u-1=(1 / 2 i) \int_{Y}^{z}\left(\mathrm{e}^{-2 \mathrm{i}(t-z)}-1\right) F(t) u(t) d t$ and we choose a path of integration on which $\operatorname{Im}(t-z) \leqq 0$. Finally we set $U=u \mathrm{e}^{-\mathrm{i} z}$.

\section{Proof of Theorem 2, first part}

Suppose that $H$ satisfies the hypotheses of the theorem, with

$$
N_{1}(r, H)=o(T(r, H)), \quad N_{1}(r, H)=O(\log r)^{2-\delta},
$$

$\delta$ being a positive constant. Then $[10,11]$ we can write, locally,

$$
H=w_{1} / w_{2}, \quad W\left(w_{1}, w_{2}\right)=1,
$$

where the $w_{j}$ are solutions of the equation

$$
w^{\prime \prime}+b(z) w=0
$$

with $b(z)=\frac{1}{2}\{H, z\}$. The function $b$ is meromorphic in the plane, while the solutions $\boldsymbol{w}_{j}$ admit analytic continuation along any path avoiding poles of $b$. For convenience later on we choose constants $\phi, \psi$ such that $H_{1}(z)=(H(z)-\phi) /(H(z)-\psi)$ has only simple zeros and poles and such that $\bar{N}\left(r, H_{1}\right) \sim T(r, H)$. Further, we write $H_{1}(z)=\sigma(z) / \tau(z)$ with $\sigma$ and $\tau$ entire functions with no common zeros, and we choose a zero $u_{0}$ of $\tau(z)$. This gives $H_{1}^{\prime}(z)=W(\tau, \sigma) \tau^{-2}=h \tau^{-2}$, with $N(r, 1 / h)=O(\log r)^{2-\delta}$, and we can assume that $T(r, h)=O(\log r)^{2-\delta}$, because otherwise we can write $h(z)=h^{*}(z) \mathrm{e}^{-2 \lambda(z)}$ with $\lambda(z)$ entire and $T\left(r, h^{*}\right)=O(\log r)^{2-\delta}$, and we need only replace $\sigma$ and $\tau$ by $\sigma \mathrm{e}^{\lambda}$ and $\tau \mathrm{e}^{\lambda}$.

Returning to the function $H$, we have 


$$
H^{\prime}=-1 / V_{2}, \quad(1 / H)^{\prime}=1 / V_{1}, \quad V_{j}=\left(w_{j}\right)^{2}
$$

and so the $V_{j}$ are meromorphic in the plane and

$$
N\left(r, V_{1}\right)+N\left(r, V_{2}\right)+N(r, b)=O\left(N_{1}(r, H)\right)=O(\log r)^{2-\delta}
$$

We can write

$$
V_{j}=g_{j} / h_{j}, \quad b=a / c, \quad T\left(r, h_{j}\right)+T(r, c)=O(\log r)^{2-\delta},
$$

with $g_{j}, h_{j}, a, c$ entire, with in each case the numerator and denominator having no common zeros. We now divide the proof into certain cases.

Case A. Suppose that $T(r, a) \neq O(\log r)^{2-\delta / 2}$.

In this case we easily obtain arbitraily large $s_{0}$ with

$$
\log M\left(s_{0}, a\right)>2\left(\log s_{0}\right)^{2-\delta / 2}, \quad v\left(s_{0}, a\right)>\left(\log s_{0}\right)^{1-\delta / 2} .
$$

By the discussion in Section 2, we can find $s_{1}, s_{2}$ satisfying

$$
s_{0} \leqq s_{1}<s_{2} \leqq\left(s_{0}\right)^{2}, \quad \log \left(s_{2} / s_{1}\right) \geqq\left(\log s_{0}\right)^{\delta / 2}
$$

and such that we have, for some non-zero constant $\gamma_{0}$,

$$
\begin{gathered}
c(z)=\gamma_{0} z^{v}(1+o(1)), \quad c^{\prime}(z) / c(z)=(v / z)(1+o(1)), \quad c^{\prime \prime}(z) / c(z)=(v / z)^{2}(1+o(1)), \\
v=n\left(s_{1}, 1 / c\right)=O\left(\log s_{0}\right)^{1-\delta}, \quad s_{1} \leqq|z| \leqq s_{2},
\end{gathered}
$$

with similar representations for $h_{1}$ and $h_{2}$ in the same annulus.

We now choose $r$, normal for the functions $a, g_{1}$ and $g_{2}$, and $z_{0}$ and constants $\gamma_{1}, \gamma$, such that $\frac{1}{4} s_{2} \leqq r \leqq \frac{1}{2} s_{2},\left|a\left(z_{0}\right)\right|=M(r, a)$, and $\frac{1}{2}<\gamma_{1}<\gamma<\frac{2}{3}$. We then have, provided $s_{0}$ was chosen large enough,

$$
a(z)=a\left(z_{0}\right)\left(z / z_{0}\right)^{v(r, a)}(1+o(1)), \quad a^{(j)}(z) / a(z)=(v(r, a) / z)^{j}(1+o(1)),
$$

for $j=1,2$ and $z=z_{0} \mathrm{e}^{\tau},|\tau|<v(r, a)^{-\gamma_{1}}$. Setting $N=v(r, a)-v=v(r, a)(1+o(1))$, using (3.7), we now have, by (3.9),

$$
b(z)=b\left(z_{0}\right)\left(z / z_{0}\right)^{N}(1+o(1)), \quad \text { for } z=z_{0} \mathrm{e}^{\mathrm{x}},|\tau| \leqq N^{-\gamma},
$$

and we note that, with $M(r, b)=\max \{|b(z)|:|z|=r\}$,

$$
\left|b\left(z_{0}\right)\right| \geqq(1-o(1)) M(r, b), \quad\left(\log s_{0}\right)^{1-\delta}<N \leqq\left(\log \left|b\left(z_{0}\right)\right|\right)^{2} .
$$

We now make the same local change of variables as in $[12$, Section 7$]$, and $[15$, 
Section 4]. We set $D(s)=\left\{z:|\log | z / z_{0}|| \leqq s N^{-\gamma},\left|\arg \left(z / z_{0}\right)\right| \leqq s N^{-\gamma}\right\}$. We write $z_{1}=$ $z_{0} \exp \left(-N^{-\gamma}\right)$ and on $D(1)$ we define branches of $b(z)^{1 / 2}$ and $z^{N / 2}$. Defining $Z$ exactly as in [12, Section 7], we obtain, in $D(1 / 2)$,

$$
\begin{aligned}
Z=b\left(z_{1}\right)^{1 / 2} z_{1} 2 /(N+2)+ & \int_{z_{1}}^{z} b(t)^{1 / 2} d t=(1+o(1)) b\left(z_{0}\right)^{1 / 2} 2 z^{(N+2) / 2} z_{0}^{-N / 2}(N+2)^{-1} \\
& =(1+o(1)) b(z)^{1 / 2} 2 z /(N+2)
\end{aligned}
$$

As in [12] we conclude that the function $Z$ has in $D(1 / 4)$ simple islands over the closed region $D^{*}$ given by

$$
|\log | Z / Z_{0}|| \leqq N^{1 / 3}, \quad|\arg Z| \leqq \pi / 4, \quad Z_{0}=\left|b\left(z_{0}\right)^{1 / 2} 2 z_{0} /(N+2)\right|,
$$

and we choose such a pre-image $D^{* *}$. By (3.12), $Z_{0} \exp \left(-N^{1 / 3}\right)$ and the minimum modulus of $b(z)$ on $D^{* *}$ are large. Indeed, we have, using (3.7),

$$
\log r+\log N=o\left(\log Z_{0}\right), \quad r=\left|z_{0}\right|
$$

As in [12, Section 8] we make the transformation $W(Z)=b(z)^{1 / 4} w(z)$, where $w$ solves (3.3), $z$ lies in $D^{* *}$ and $Z$ in $D^{*}$. The equation (3.3) transforms to

$$
d^{2} W / d Z^{2}+\left(1-F_{0}(Z)\right) W=0, \quad F_{0}(Z)=b^{\prime \prime}(z) / 4 b(z)^{2}-5 b^{\prime}(z)^{2} / 16 b(z)^{3},
$$

in which, using (3.7), (3.9), (3.10) and (3.13), $\left|F_{0}(Z)\right| \leqq c_{4}|Z|^{-2}$ in $D^{*}$. By Lemma 1 of [12] there exist solutions $U, V$ of $(3.16)$ in $D^{*}$ such that

$$
U(Z)=(1+o(1)) \mathrm{e}^{-\mathrm{i} Z}, \quad V(Z)=(1+o(1)) \mathrm{e}^{\mathrm{i} Z}, \quad W(U, V)=2 i+o(1) .
$$

We can write, in $D^{* *}$, for some constants $A, B, C, D$,

$$
u(z)=b(z)^{-1 / 4} U(Z), \quad v(z)=b(z)^{-1 / 4} V(Z), \quad w_{1}=A u+B v, \quad w_{2}=C u+D v .
$$

We estimate $A, B, C$ and $D$, using a method different to that of [12]. Since

$$
-b=w_{j}^{\prime \prime} / w_{j}=\frac{1}{2}\left(g_{j}^{\prime \prime} / g_{j}-h_{j}^{\prime \prime} / h_{j}\right)-\frac{1}{4}\left(g_{j}^{\prime} / g_{j}\right)^{2}+\frac{3}{4}\left(h_{j}^{\prime} / h_{j}\right)^{2}-\frac{1}{2}\left(g_{j}^{\prime} / g_{j}\right)\left(h_{j}^{\prime} / h_{j}\right),
$$

we obtain, denoting absolute constants by $c_{j}$ and using $(3.9), v\left(r, g_{j}\right) \leqq c_{1} r M(r, b)^{1 / 2}$ and so

$$
\log M\left(r, g_{j}\right)+\log M\left(r, g_{j}^{\prime}\right) \leqq \log M_{0}=c_{2} r(\log r) M(r, b)^{1 / 2}
$$

Therefore we have, for $s_{1} \leqq|z| \leqq r$, using (3.7),

$$
\log \left(\left|w_{j}(z)\right|+\left|w_{j}^{\prime}(z)\right|\right) \leqq \log M_{0}+O(\log r)^{2-\delta} \leqq \log M_{1}=c_{3} r(\log r) M(r, b)^{1 / 2} .
$$


To estimate $A, B, C, D$ we note that, in $D^{* *}$,

$$
w_{1}=A u+B v, \quad w_{1}^{\prime}=A u^{\prime}+B v^{\prime}, \quad W(u, v)=2 i+o(1) .
$$

We choose $Z^{*}$ in $D^{*}$ with $\left|Z^{*}\right| \leqq Z_{0} / 2$ and $\left|U\left(Z^{*}\right)\right| \leqq 2,\left|U^{\prime}\left(Z^{*}\right)\right| \leqq 2$. Let $z^{*}$ be the preimage of $Z^{*}$ in $D^{* *}$, so that $\left|z^{*}\right| \leqq r$ and $\left|u\left(z^{*}\right)\right| \leqq 2$ and $\left|u^{\prime}\left(z^{*}\right)\right| \leqq 2 M(r, b)^{1 / 2}$. Now (3.22) gives $B=W\left(u, w_{1}\right) W(u, v)^{-1}$ so that

$$
|B| \leqq 2 M_{1} M(r, b)^{1 / 2} \leqq \exp \left(c_{4}(\log r) r M(r, b)^{1 / 2}\right),
$$

and the same estimate holds for $A, C$ and $D$. Further,

$$
1=W\left(w_{1}, w_{2}\right)=(A D-B C) W(u, v)=(A D-B C)(2 i+o(1))
$$

Thus, in any pair $\{A, B\},\{A, C\},\{B, D\},\{C, D\}$, each term has modulus at most

$$
M=\exp \left(c_{5} N Z_{0} \log r\right), \quad \log M=o\left(\exp \left(N^{1 / 4}\right) Z_{0}\right),
$$

while at least one term has modulus at least $1 / M$.

\section{Completion of the proof of Theorem 2, in Case $A$}

We choose a small positive constant $\eta$, and we write

$$
\begin{gathered}
g(z)=w_{2} z-w_{1}=F_{1} u+F_{2} v, \quad F_{1}(z)=C z-A, \quad F_{2}(z)=D z-B, \\
G(Z)=b(z)^{1 / 4} g(z), \quad P_{j}(Z)=F_{j}(z) .
\end{gathered}
$$

Now (3.23) implies that either $|A D| \geqq 1 / 6$ or $|B C| \geqq 1 / 6$. In this section we use $D_{j}$ to denote positive constants not depending on $\eta$. It is easy to see that we have

$$
\begin{gathered}
|1-C z / A| \geqq\left(D_{1}(N+2)\right)^{-1},|1-B / D z| \geqq\left(D_{1}(N+2)\right)^{-1}, \\
\left|F_{j}^{\prime}(z) / F_{j}(z)\right| \leqq D_{1}(N+2) /|z|,
\end{gathered}
$$

if $A D \neq 0$ and, if $C D \neq 0$,

$$
\begin{gathered}
|1-D z / B| \geqq\left(D_{1}(N+2)\right)^{-1},|1-A / C z| \geqq\left(D_{1}(N+2)\right)^{-1}, \\
\left|F_{j}^{\prime}(z) / F_{j}(z)\right| \leqq D_{1}(N+2) /|z|,
\end{gathered}
$$

outside at most four discs each of radius at most $D_{1} r(N+2)^{-1}$. Further, these discs have images in the $Z$-plane which each lie in an annulus of form $R_{1} \leqq|Z| \leqq R_{2}$ with $\log \left(R_{2} / R_{1}\right) \leqq D_{2}$. This easily gives us $d \geqq 1 / 2$ such that 


$$
\left|P_{j}^{\prime}(Z) / P_{j}(Z)\right| \leqq D_{3} / Z\left|, d N^{1 / 3} \leqq \log \right| Z / Z_{0}\left|\leqq(d+1 / 12) N^{1 / 3},\right| \arg Z \mid \leqq \pi / 4
$$

and such that we have (4.2) and/or (4.3) in the pre-image $D^{\prime}$ in $D^{* *}$ of the region in (4.4). Now suppose that $z \in D^{\prime}$ is a zero of $g$. Then we have $H(z)=z$, since the $w_{j}$ have no common zeros, and $\mu=v / u=(A-C z) /(D z-B)$ so that

$$
-1 / H^{\prime}=\left(w_{2}\right)^{2}=u v \mu^{-1}(C+D \mu)^{2}=u v(D z-B)^{-1}(A-C z)^{-1}(A D-B C)^{2}
$$

If $|A D| \geqq 1 / 6$ this is, using (3.12), (3.13), (3.15), (3.23) and (4.2),

$$
\begin{gathered}
(-1 / 4+o(1)) b(z)^{-1 / 2}(A D z(1-B / D z)(1-C z / A))^{-1}=O\left(|b(z)|^{-1 / 2}|z|^{-1}(N+2)^{2}\right) \\
=O\left((N+2)|Z|^{-1}\right)=O\left(Z_{0}^{-1}\right)=o\left(|z|^{-q}\right) .
\end{gathered}
$$

We argue similarly if $|B C| \geqq 1 / 6$. Thus the conclusion of Theorem 2 holds in Case $A$, provided that we can show that $D^{\prime}$ contains a zero of $g$.

To prove the last assertion, we assume that $D^{\prime}$ contains no zero of $g$. In the region in (4.4) we have, using (3.15), (3.24), the last remark in Section 3 and (4.2) or (4.3)

$$
|\log | P_{j}(Z)|| \leqq D_{4}(\log M+\log r+\log (N+2)) \leqq o(|Z|)
$$

and $\log ^{+}|G(Z)|=O(|Z|)$. Further, we have, provided $s_{0}$ was chosen large enough,

$$
|\log | \mathrm{e}^{\mathrm{i} Z}|| \geqq D_{5}|Z||\arg Z| \geqq D_{5} \eta \exp \left(\frac{1}{2} N^{1 / 3}\right) Z_{0}, \quad \text { for } \pi / 4 \geqq|\arg Z| \geqq \eta
$$

so that $|G(Z)|>1$ on $\arg Z=\eta$, and we obtain, arguing as in $[12],\left|G^{\prime}(Z) / G(Z)\right| \leqq D_{6}$ in the region $(d+1 / 48) N^{1 / 3} \leqq|\log | Z / Z_{0}|| \leqq(d+1 / 16) N^{1 / 3},|\arg Z| \leqq \pi / 16$. We apply the argument principle to $G$ on the boundary of the region

$$
m_{1}=(d+1 / 48) N^{1 / 3} \leqq|\log | Z / Z_{0} \| \leqq m_{2}=(d+1 / 16) N^{1 / 3},|\arg Z| \leqq \eta
$$

On the straight line segments which form part of this boundary we have

$$
G(Z)=(1+o(1)) U(Z) P_{1}(Z), \arg Z=\eta, \text { and } G(Z)=(1+o(1)) V(Z) P_{2}(Z), \arg Z=-\eta
$$


The change in $\log G(Z)$ as $Z$ describes once counter-clockwise the boundary of the region in (4.5) is then, using (4.4),

$$
2 \mathrm{i} \cos (\eta) Z_{0}\left(\mathrm{e}^{m_{2}}-\mathrm{e}^{m_{1}}\right)+O\left(\eta Z_{0} \mathrm{e}^{m_{2}}\right)+O\left(\left(m_{2}-m_{1}\right)\right) \neq 0
$$

\section{Proof of Theorem 2: Case $B$}

Here we suppose that $T(r, a)=O(\log r)^{2-\delta / 2}$. By (3.4) and (3.6), we must have $\rho\left(g_{j}\right)=\rho(H)$ for each $j$. We choose $q^{\prime}$ with $q<q^{\prime}<\rho(H)$ and $\varepsilon>0$ with $(1+\varepsilon) q<q^{\prime}$, and we can find arbitrarily large $s_{0}$ such that, for some $j$,

$$
v\left(s_{0}, g_{j}\right)>\left(s_{0}\right)^{q^{\prime}} .
$$

Since $a$ and $c$ both have small growth we can find $s_{1}, s_{2}$ such that

$$
s_{0} \leqq s_{1} \leqq\left(s_{0}\right)^{1+\varepsilon / 2}, \log \left(s_{2} / s_{1}\right)=\left(\log s_{0}\right)^{8 / 5}
$$

and such that in the annulus $s_{1} \leqq|z| \leqq s_{2}$ we have

$$
b(z)=\alpha z^{N}(1+\varepsilon(z)), \varepsilon(z)=o(1), \varepsilon^{\prime}(z)=o(1) / z, \varepsilon^{\prime \prime}(z)=o(1) / z^{2}
$$

with $\alpha$ a non-zero constant (depending on the annulus) and $N=n\left(s_{1}, 1 / a\right)-n\left(s_{1}, 1 / c\right)$, and

$$
h_{j}^{(m)}(z) / h(z)=O\left(n\left(s_{1}, 1 / h_{j}\right) /|z|\right)^{m}
$$

for $j=1,2, m=1,2$. We remark that at this point the second condition of (1.4) seems to be necessary for our method to work, because if the entire functions $a$ and $c$ are of roughly the same growth we have otherwise apparently no information on the local behaviour of $a / c$. We then have, by (3.19) and (5.1), if $r \in\left[s_{1}, s_{2}\right]$ is normal for both $g_{j}$, and $z$ is a maximum modulus point of $g_{j}$ with $|z|=r$,

$$
v\left(r, g_{j}\right)^{2}=(-4+o(1)) \alpha z^{N+2},
$$

and it follows that since $v\left(r, g_{j}\right)$ is increasing we must have $N \geqq-2$ in (5.3). Further, $|\alpha|^{1 / 2} r^{(N+2) / 2}$ must be large, if $r$ is normal for the $g_{j}$. We postpone the case $N=-2$ to Section 6 and assume for the remainder of this section that $N \geqq-1$. We write $\alpha=\beta^{2}$ and we choose a real $\theta_{0}$ such that $\arg \beta+(N+2) \theta_{0} / 2=0(\bmod 2 \pi)$. We set $z_{1}=s_{1} \mathrm{e}^{\mathrm{i} \theta_{0}}$, and we make the change of variables

$$
\begin{gathered}
Z=2 \beta(N+2)^{-1}\left(z_{1}\right)^{(N+2) / 2}+\int_{z_{1}}^{z} b(t)^{1 / 2} d t=(2+o(1)) \beta(N+2)^{-1} z^{(N+2) / 2}, \\
\text { for } s_{1} \leqq|z| \leqq s_{2},\left|\arg z-\theta_{0}\right|<\pi,
\end{gathered}
$$


using (5.3). Now the function $Z$ maps a sub-domain $D^{* *}$ of the annulus $4 s_{1} \leqq|z| \leqq s_{2}$ univalently onto the region

$$
\begin{gathered}
T_{1}=2|\beta|(N+2)^{-1}\left(8 s_{1}\right)^{(N+2) / 2} \leqq|Z| \leqq T_{2}=2|\beta|(N+2)^{-1}\left(s_{2} / 2\right)^{(N+2) / 2} \\
|\arg Z| \leqq \pi / 4
\end{gathered}
$$

and $T_{1}$ must be large, by (5.2) and the remark following (5.5). The same transformation $W(Z)=b(z)^{1 / 4} w(z)$ as in Section 3 gives (3.16) again, in which $F_{0}(Z)=O\left(|Z|^{-2}\right)$, by (5.3). Again, we obtain the solutions $U, V$ as in (3.17) in the region (5.7), and we estimate the coefficients $A, B, C, D$. We choose $r$ with $12 s_{1} \leqq r \leqq 16 s_{1}$, normal for both the $g_{j}$, and we obtain (3.20) again. Using the remark following (5.5), this leads to (3.21), valid for $|z|=r$. We choose $Z^{*}$ in the region (5.9) with $\left|U\left(Z^{*}\right)\right| \leqq 2,\left|U^{\prime}\left(Z^{*}\right)\right| \leqq 2$, and with pre-image $z^{*}$ in $D^{* *}$ satisfying $\left|z^{*}\right|=r$. We then have

$$
\left|u\left(z^{*}\right)\right| \leqq 2\left|b\left(z^{*}\right)\right|^{-1 / 4} \leqq 4|\alpha|^{-1 / 4}\left|z^{*}\right|^{-(N+2) / 4}\left|z^{*}\right|^{1 / 2} \leqq o\left(\left|z^{*}\right|^{1 / 2}\right)
$$

using (5.5). Further, using (5.3),

$$
\left|u^{\prime}\left(z^{*}\right)\right| \leqq o\left(\left|z^{*}\right|^{1 / 2}\right)\left(\left|b\left(z^{*}\right)\right|^{1 / 2}+\left|b^{\prime}\left(z^{*}\right) / 4 b\left(z^{*}\right)\right|\right) \leqq o\left(|\beta| r^{(N+2) / 2}+(N+2)\right) .
$$

This reasoning shows that the coefficients $A, B, C, D$ all have modulus at most $M$, where

$$
\log M=\log (N+2)+d_{1}|\beta|\left(16 s_{1}\right)^{(N+2) / 2} \log \left(16 s_{1}\right),
$$

denoting constants by $d_{j}$. We now write (4.1) again and we have (4.2) and/or (4.3) for all $z$ in $D^{* *}$ outside at most four discs whose images each lie in an annulus in the $Z$-plane of logarithmic measure at most $d_{2}$. Since $\log \left(T_{2} / T_{1}\right) \geqq d_{3}(N+2)\left(\log s_{0}\right)^{\delta / 5}$, we can choose $S_{1}, S_{2}$ such that

$$
\log T_{1}+d_{4}(N+2)\left(\log s_{0}\right)^{\delta / 5} \leqq \log S_{1}<\log S_{2}=\log S_{1}+d_{4}(N+2)\left(\log S_{0}\right)^{\delta / 5} \leqq \log T_{2},
$$

and such that we have (4.2) and/or (4.3) in the pre-image in $D^{* *}$ of the region $S_{1} \leqq|Z| \leqq S_{2},|\arg Z| \leqq \pi / 4$. We also have

$$
(\log M) / S_{1} \leqq o(1)+d_{5} 2^{(N+2) / 2}(N+2) \log \left(16 s_{1}\right) \exp \left(-d_{4}(N+2)\left(\log s_{0}\right)^{8 / 5}\right)=o(1)
$$

Therefore we have

$$
\begin{gathered}
\log M=o(|Z|),|\log | P_{j}(Z) \|=o(|Z|),\left|P_{j}^{\prime}(Z) / P_{j}(Z)\right| \leqq d_{6} /|Z|, \\
\text { for } S_{1} \leqq|Z| \leqq S_{2},|\arg Z| \leqq \pi / 4 .
\end{gathered}
$$

We argue as in Section 4, assuming again that $\eta$ is a small positive constant. If the region (5.10) contains a zero of $G(Z)$ then at the pre-image $z$ in $D^{* *}$ we have 


$$
\begin{aligned}
\left|1 / H^{\prime}(z)\right| & \leqq d_{7}(N+2)^{2}|b(z)|^{-1 / 2}|z|^{-1} \leqq d_{8}(N+2)^{2}|\beta|^{-1}\left(2 s_{1}\right)^{-(N+2) / 2}\left(|z| / 2 s_{1}\right)^{-(N+2) / 2} \\
& \leqq d_{9} v\left(s_{0}, g_{j}\right)^{-1}(N+2)^{2} 2^{-(N+2) / 2}=O\left(\left(s_{0}\right)^{-q^{\prime}}\right)=o\left(|z|^{-q}\right)
\end{aligned}
$$

using (5.1), (5.2), (5.5) and (5.9). To show that such a fixpoint of $H$ exists, if $s_{0}$ is large enough, we suppose that the region in (5.10) contains no zero of $G(Z)$. Then $G(Z)$ is large on $\arg Z=\eta$, by $(5.10)$, and $\left|G^{\prime}(Z) / G(Z)\right| \leqq d_{10}$ for $2 S_{1} \leqq|Z| \leqq S_{2} / 2,|\arg Z| \leqq \pi / 64$. Thus the change in $\log G(Z)$ as $Z$ describes once counter-clockwise the boundary of the region $2 S_{1} \leqq|Z| \leqq S_{2} / 2$, $|\arg Z| \leqq \eta$ is

$$
2 \mathrm{i}\left((1 / 2) S_{2}-2 S_{1}\right) \cos (\eta)+O\left(\eta S_{2}\right)+O\left(\log \left(S_{2} / S_{1}\right)\right) \neq 0 .
$$

\section{The case where $N=-2$ in (5.3)}

At this stage we recall the function $H_{1}$ defined following (3.3), to which we will apply an argument similar to that of [18]. By (5.5) and the discussion in Section 2, we can write $b(z)^{1 / 2}=\mathrm{i} L z^{-1}(1+\omega(z))$, with $L$ large and positive and $|\omega(z)| \leqq$ $O\left(\exp \left(-\left(\log s_{0}\right)^{\delta / 10}\right)\right)$. As $L$ is large, we can again use the transformation $w(z)=$ $b(z)^{-1 / 4} W(Z)$, where

$$
Z=\mathrm{i} L \log s_{1}+\int_{s_{1}}^{z} b(t)^{1 / 2} d t=\mathrm{i} L \log z+o(L), s_{1} \leqq|z| \leqq s_{2},|\arg z| \leqq 4 \pi,
$$

mapping onto a region containing the rectangle

$$
|\operatorname{Re}(Z)| \leqq 3 \pi L, L \log \left(2 s_{1}\right) \leqq \operatorname{Im}(Z) \leqq L \log \left(s_{2} / 2\right) .
$$

The equation (3.3) transforms to (3.16) again, with this time $\left|F_{0}(Z)\right| \leqq C_{1} L^{-2}$, denoting absolute constants by $C_{j}$, and we note that, by (5.1) and (5.5), we have $L>\left(s_{1}\right)^{q}$. Lemma 1 gives us solutions $U, V$ of (3.21) in the region (6.1) satisfying $U(Z) \sim \mathrm{e}^{-\mathrm{i} Z}, V(Z) \sim \mathrm{e}^{\mathrm{i} Z}$. This gives us, on multiplying by a constant, solutions $u, v$ of (3.3) satisfying

$$
u(z)=z^{L+1 / 2} \mathrm{e}^{o(L)}, \quad v(z)=z^{-L+1 / 2} \mathrm{e}^{o(L)},
$$

for $4 s_{1} \leqq|z| \leqq s_{2} / 4,|\arg z| \leqq \pi$, and there are constants $A, B$, not both zero, such that $H_{1}^{\prime}(z)=h(z) \tau(z)^{-2}=(A u(z)+B v(z))^{-2}$. We assert the existence of $R$ such that, for some non-zero constant $\gamma_{2}$,

$$
H_{1}^{\prime}(z)=\gamma_{2} z^{-1 \pm 2 L} \mathrm{e}^{o(L)} \quad \text { for } \quad 4 s_{1} \leqq \frac{1}{4} R \leqq|z| \leqq 8 R \leqq s_{2} / 4 \text {, }
$$

and such that $|h(z)| \geqq 1$ on $|z|=R$. If $A B=0$, this is obvious. Otherwise, we choose $R$ such that $|h(z)| \geqq 1$ on $|z|=R$ and $|\log R-(1 / 2 L) \log | B / A \|>2$ and (6.2) follows, as $L$ is large. Since $n\left(r, 1 / H_{1}^{\prime}\right)=O(\log r)^{1-\delta},(5.1),(5.5)$ and (6.2) give 


$$
2 L \sim n\left(R / 2, H_{1}^{\prime}\right) \leqq C_{2} N(R, 1 / \tau) \leqq C_{2} \log M(R, \tau)+C_{3} .
$$

Therefore (6.2) gives $\log M(R, \tau) \sim \log m_{0}(R, \tau)$, with $m_{0}(R, \tau)$ denoting the minimum modulus. We choose a fixed $z_{2}$ with $\left|z_{2}\right|=R$. Integrating $h \tau^{-2}$ around $|z|=R$ we obtain $\left|\sigma(z) / \tau(z)-\sigma\left(z_{2}\right) / \tau\left(z_{2}\right)\right| \leqq R M(R, h) m_{0}(R, \tau)^{-2}$ and so

$$
\left|\sigma(z) \tau\left(z_{2}\right)-\sigma\left(z_{2}\right) \tau(z)\right| \leqq R M(R, h) m_{0}(R, \tau)^{-2} M(R, \tau)\left|\tau\left(z_{2}\right)\right|
$$

for $|z| \leqq R$. Applying this inequality with $z=u_{0}$, the zero of $\tau(z)$ fixed in Section 3, we obtain $m_{0}(R, \tau)^{2} \leqq C_{4} R M(R, h) M(R, \tau)$, a contradiction.

\section{Proof of Theorem 1}

We shall prove parts (i) and (ii) simultaneously, and we denote non-integrated counting functions, as usual, by $n(r)$. As in [2], we use the auxiliary function

$$
H=z-h f / f^{\prime}, h=1 /(1-a), H^{\prime}=h\left(f f^{\prime \prime}-a f^{\prime 2}\right) /\left(f^{\prime 2}\right) .
$$

Now multiple poles of $H$ can only occur at zeros of $f^{\prime}$ of multiplicity $m \geqq 2$ which are not also zeros of $f$, and they contribute $m-1$ to $n_{1}(r, H)$ and the same to $n_{2}(r)$ and $n_{3}(r)$. Further, zeros of $H^{\prime}$ cannot occur at poles of $f$, since $a$ is not of the form $(m+1) / m$ with $m$ a positive integer, and with the hypotheses of part (ii) they cannot occur at multiple zeros of $f$ either. Therefore, we have $N\left(r, 1 / H^{\prime}\right) \leqq N(r)$ in both cases. However, at any fixpoint of $H$ we clearly have $f=0$ or $f=\infty$, and $H^{\prime}=O(1)$.

Thus $H$ must be a rational function, with no multiple points, and so a Möbius transformation, and we obtain $f^{\prime}(z) / f(z)=h(C z+D) /\left(C z^{2}+(D+S) z+T\right)$, with $C, D, S, T$ constants, and since $f$ is transcendental we see at once that $C$ and $D+S$ must vanish.

\section{A better estimate when $f^{\prime} / f$ has large growth}

Suppose that $H$ is transcendental meromorphic in the plane, such that $\log ^{+}\left|H^{\prime}(z)\right| \leqq$ $O(\log |z|)$ at all fixpoints $z$ of $H$ with $|z|$ large, and suppose that $N_{1}(r, H)=O\left(r^{m}\right)$, with $m$ a positive constant. It seems reasonable to believe that these hypotheses might imply that $H$ has finite order. Our method does not appear to give this, but we can write (3.2), (3.3), (3.4) and (3.6) with $T\left(r, h_{j}\right)+T(r, c)=O\left(r^{2 m}\right)$. Suppose that $\rho(a)$ is large compared to $m$. Choosing $\gamma \in(1 / 2,2 / 3)$, we can find arbitrarily large $r$, normal for $a$ and the $g_{j}$, such that $(\log N) /(m \log r)$ is large, where $N=v(r, a)$, and such that, choosing $z_{0}$ with $\left|z_{0}\right|=r$ and $\left|a\left(z_{0}\right)\right|=M(r, a)$, we have (3.10), and

$$
\left|c^{\prime}(z) / c(z)\right|+|\log | c(z) \| \leqq r^{d_{1} m} \quad \text { for } \quad|| z|-r| \leqq r^{-d_{2} m},
$$

with similar estimates for the $h_{j}$, and with $d_{j}$ denoting constants not depending on $m$. This implies that (3.11) holds, and we have 


$$
\log \mid b\left(z_{0}|\geqq \log | a\left(z_{0}\right)\left|-O\left(r^{d_{1} m}\right) \sim \log \right| a\left(z_{0}\right) \mid, \quad N \leqq\left(\log \left|b\left(z_{0}\right)\right|\right)^{2} .\right.
$$

Applying the same change of variables as in Section 3, we have, in particular, (3.15). The estimate (3.20) holds for the $g_{j}$, and we obtain (3.21), for $r-r^{-d_{2} m} \leqq|z| \leqq r$, and with $(\log r)^{2-\delta}$ replaced by $r^{d_{1} m}$. The coefficients $A, B, C, D$ all have modulus at most

$$
\exp \left(\exp \left(r^{d_{3} m}\right) r\left|b\left(z_{0}\right)\right|^{1 / 2}\right) \leqq M=\exp \left(\exp \left(N^{1 / 12}\right) Z_{0}\right)
$$

The argument of Section 4 goes through, since $\log M=o(|Z|)$ for $Z$ satisfying (4.4), and we obtain fixpoints $z$ of $H$ with $H^{\prime}$ large, contradicting our initial assumption. Hence the order of $a$ can be bounded in terms of $m$. Estimating the $g_{j}$ using (3.19), we have the following.

Theorem 3. Suppose that $0<m<\infty$ and $f$ is meromorphic in the plane, and that $N(r)$ counts the zeros of $f f^{\prime \prime}$ which are not multiple zeros of $f$. If $N(r)=O\left(r^{m}\right)$, then $\log T\left(r, f^{\prime} / f\right)=O\left(r^{n}\right)$, the positive constant $n$ depending only on $m$.

\section{REFERENCES}

1. J. M. Anderson, I. N. Baker and J. Clunie, The distribution of values of certain entire and meromorphic functions, Math. Z. 178 (1981), 509-525.

2. W. BergweILer, On the zeros of certain homogeneous differential polynomials, Arch. Math. (Basel) 64 (1995), 199-202.

3. W. Bergweiler and A. ERemenko, On the singularities of the inverse to a meromorphic function of finite order, Rev. Mat. Iberoamericana, to appear.

4. A. Eremenko, Meromorphic functions with small ramification, Indiana Univ. Math. J. 42 (1994), 1193-1218.

5. G. Frank, Eine Vermutung von Hayman über Nullstellen meromorpher Funktionen, Math. Z. 149 (1976), 29-36.

6. G. Frank and S. Hellerstein, On the meromorphic solutions of nonhomogeneous linear differential equations with polynomial coefficients, Proc. London Math. Soc. (3) 53 (1986), 407-428.

7. G. Frank, W. Hennekemper and G. Polloczek, Über die Nullstellen meromorpher Funktionen and ihrer Ableitungen, Math. Ann. 225 (1977), 145-154.

8. W. K. Hayman, Picard values of meromorphic functions and their derivatives, Ann. of Math. 70 (1959), 9-42.

9. W. K. Hayman, The local growth of power series: a survey of the Wiman-Valiron method, Canad. Math. Bull. 17 (1974), 317-358.

10. E. Hille, Ordinary Differential Equations in the Complex Domain (Wiley, New York, 1976).

11. I. Laine, Nevanlinna theory and complex differential equations (de Gruyter Studies in Math. 15, Walter de Gruyter, Berlin/New York 1993).

12. J. K. Langley, Proof of a conjecture of Hayman concerning $f$ and $f^{\prime \prime}, J$. London Math. Soc. (2) 48 (1993), 500-514.

13. J. K. LANGLey, On the fixpoints of composite entire functions of finite order, Proc. Roy. Soc. Edinburgh 124A (1994), 995-1001. 
14. J. K. Langley, On the multiple points of certain meromorphic functions, Proc. Amer. Math. Soc., to appear.

15. J. K. LANGley, On second order linear differential polynomials, Resultate Math. 26 (1994), 51-82.

16. E. Mues, Über eine Vermutung von Hayman, Math. Z. 119 (1972), 11-20.

17. M. Reinders, On the zeros of $W\left(g, g^{\prime}, \ldots, g^{(n-1)}\right)$, preprint.

18. D. SHEA, On the frequency of multiple values of a meromorphic function of small order, Michigan Math. J. 32 (1985) 109-116.

UNIVERSITY OF NOTTINGHAM

NotTingham

NG7 2RD

E-mail: jkl@maths.nott.ac.uk. 